\title{
Species Distribution of Candidemia and Their Susceptibility in a Single Japanese University Hospital: Prior Micafungin Use Affects the Appearance of Candida parapsilosis and Elevation of Micafungin MICs in Non-parapsilosis Candida Species
}

\author{
Yasutaka Sakamoto ${ }^{1,2}$ D, Kazuhiro Kawabe ${ }^{2}$, Tomoyo Suzuki ${ }^{2}$, Kayoko Sano ${ }^{3}$, Kazuo Ide ${ }^{2}$, Tetsuta Nishigaki ${ }^{2}$, \\ Yuki Enoki ${ }^{1, * \mathbb{D}}$, Kazuaki Taguchi ${ }^{1}$, Hirofumi Koike ${ }^{2}$, Hideaki Kato ${ }^{4}$ (D), Yukiko Sahashi ${ }^{2}$ \\ and Kazuaki Matsumoto ${ }^{1}$ (D) \\ check for \\ updates \\ Citation: Sakamoto, Y.; Kawabe, K.; \\ 1 Division of Pharmacodynamics, Keio University Faculty of Pharmacy, Tokyo 105-8512, Japan; \\ yasutaka@yokohama-cu.ac.jp (Y.S.); taguchi-kz@pha.keio.ac.jp (K.T.); matsumoto-kz@pha.keio.ac.jp (K.M.) \\ 2 Department of Pharmacy, Yokohama City University Hospital, Yokohama 236-0004, Japan; \\ k_kawabe@yokohama-cu.ac.jp (K.K.); koyama_t@yokohama-cu.ac.jp (T.S.); kazuo_i@yokohama-cu.ac.jp (K.I.); \\ ntetsuta@yokohama-cu.ac.jp (T.N.); hi00_k@yokohama-cu.ac.jp (H.K.); ysahashi@yokohama-cu.ac.jp (Y.S.) \\ 3 Department of Clinical Laboratory, Yokohama City University Hospital, Yokohama 236-0004, Japan; \\ ksano@yokohama-cu.ac.jp \\ 4 Infection Prevention and Control Department, Yokohama City University Hospital, \\ Yokohama 236-0004, Japan; ekato@yokohama-cu.ac.jp \\ * Correspondence: enoki-yk@pha.keio.ac.jp; Tel.: +81-03-5400-2656
} Suzuki, T.; Sano, K.; Ide, K.; Nishigaki, T.; Enoki, Y.; Taguchi, K.; Koike, H.; Kato, H.; et al. Species Distribution of Candidemia and Their Susceptibility in a Single Japanese University Hospital: Prior Micafungin Use Affects the Appearance of Candida parapsilosis and Elevation of Micafungin MICs in Non-parapsilosis Candida Species. J. Fungi 2021, 7, 596. https://doi.org/10.3390/jof7080596

Academic Editor: David S. Perlin

Received: 25 June 2021

Accepted: 21 July 2021

Published: 23 July 2021

Publisher's Note: MDPI stays neutral with regard to jurisdictional claims in published maps and institutional affiliations.

Copyright: (c) 2021 by the authors. Licensee MDPI, Basel, Switzerland. This article is an open access article distributed under the terms and conditions of the Creative Commons Attribution (CC BY) license (https:// creativecommons.org/licenses/by/ $4.0 /)$.
Abstract: Introduction: Micafungin is a recommended echinocandin antifungal agent for candidemia treatment and prophylaxis. However, overuse of echinocandin antifungals may cause resistance. There is currently no information available regarding the low susceptibility associated with using micafungin. This study investigated the effect of micafungin use on changes in the detected Candida species and low susceptibility. Methods: We conducted a retrospective survey and included records of Candida spp. detected in blood cultures from January 2010 to December 2018 in our hospital. Survey items included clinical outcomes at 30 days after positive cultures, patient characteristics, and drug prescription status. Patient background information included gender, previous hospitalization, stay in the intensive care unit, comorbidities, and history of surgery (within 90 days before candidemia onset) and drug exposure. Species detected and their minimum inhibitory concentrations (MICs) and amount of antifungal prescriptions by department were investigated. Risk factors for detecting C. parapsilosis and for low susceptibility to micafungin were evaluated using multivariate analysis. Results: A total of 153 Candida clinical blood isolates were collected and C. albicans was the most prevalent species, followed by C. parapsilosis and C. glabrata. In the analysis by department, antifungal use and non-albicans Candida species were most frequently detected in the hematology department. Multivariate analysis showed that prior micafungin use increased the risk of C. parapsilosis (odds ratio (OR) 4.22; 95\% confidence interval (CI) 1.39-12.79; $p=0.011$ ). $\mathrm{MIC}_{90}$ of micafungin on C. glabrata and C. parapsilosis was $1.0 \mu \mathrm{g} / \mathrm{mL}$. Prior micafungin use was clarified as a risk factor resulting in MIC $>0.06 \mu \mathrm{g} / \mathrm{mL}$ for micafungin in non-parapsilosis Candida species (OR 13.2; 95\% CI 3.23-54.2; $p<0.01$ ). Conclusion: Prior micafungin use increased the risk of $C$. parapsilosis and the MIC $>0.06 \mu \mathrm{g} / \mathrm{mL}$ of micafungin in non-parapsilosis Candida species. Since there are only a few antifungal options, further antifungal stewardship considering azole antifungal agents use is required.

Keywords: candidemia; risk factor; micafungin; C. parapsilosis; non-albicans Candida species; minimum inhibitory concentrations; low susceptibility; antifungal prescription; prior antifungal use 


\section{Introduction}

Candidemia is one cause of nosocomial bloodstream infections [1]. The mortality rate from candidemia was 39.3\% in Japan [2], 38-39.2\% in the United States [3,4], and 19-38.8\% in Europe $[5,6]$ with a higher mortality rate than other nosocomial bloodstream infections in the world. Therefore, adequately treating candidemia is critical for improving patient prognosis $[7,8]$.

Micafungin is an echinocandin antifungal agent used for the treatment and prevention of candidemia. The Infectious Diseases Society of America guidelines recommend micafungin, caspofungin, and anidulafungin as the initial therapy for non-neutropenic and neutropenic patients [9]. The European Society of Clinical Microbiology and Infectious Diseases guidelines recommend micafungin for the initial treatment of candidemia in non-neutropenic and neutropenic patients [10] and also for prophylaxis against allogeneic hematopoietic stem cell recipients [11]. In the Japanese Domestic Guidelines for Management of Deep-seated Mycosis 2014, micafungin is recommended as an initial treatment for candidemia in severe non-neutropenic and neutropenic patients [12,13]. Micafungin is also recommended for prophylaxis after allogeneic hematopoietic stem cell transplantation in the Prevention and Treatment of Fungal Infections guidelines of the Japan Society for Hematopoietic Cell Transplantation [14]. Due to their higher fungicidal activity compared to other antifungals, such as azoles [9] and fewer side effects and interactions [15], echinocandin antifungals, including micafungin, are frequently used in daily practice for those at a higher risk and severity of Candida infection after onset. Furthermore, micafungin is more frequently used than caspofungin in Japan [16]. Micafungin was marketed approximately 6 years earlier than caspofungin and there is an associated brand familiarity for this antifungal agent.

In a survey of detected Candida species in blood cultures, the percentage of detected Candida species in Japan was the highest for C. albicans, followed by C. parapsilosis and C. glabrata [17-19]. In the USA, C. albicans is also ranked first; however, this is followed by C. glabrata [20]. Forrest et al. studied the use of caspofungin and the frequency of detection of C. parapsilosis [21], and high micafungin use was considered one reason for the high frequency of detection of $C$. parapsilosis in Japan. To prove this hypothesis, it is necessary to examine whether similar results can be obtained in Japan, where micafungin use is high. No previous studies have investigated whether prior micafungin use is a risk factor for $C$. parapsilosis detection. In recent years, there are studies on the resistance of non-parapsilosis Candida species to echinocandin antifungals [22]. It is helpful to promote antifungal stewardship to clarify the current status of non-parapsilosis Candida species low susceptibility by prior administration of micafungin.

The present study investigated the causative species of candidemia and their drug susceptibility, and the use of antifungal agents. Risk factors associated with detecting C. parapsilosis and increasing the minimum inhibitory concentration (MIC) of nonparapsilosis Candida species were also investigated.

\section{Materials and Methods}

\subsection{Ethics}

This study was approved by the Yokohama City University Ethics Committee (approval number: B190600046, 8 August 2019).

\subsection{Patients and Episode}

Records from the microbiology laboratory were evaluated to identify patients with positive peripheral blood cultures (including central venous (CV) catheters) for Candida spp. from January 2010 to December 2018. Isolation of Candida spp. from at least one blood culture of a patient was defined as candidemia. If the same species was detected two times or more in the same patient, only the first time was included in the analysis.

Survey items included clinical outcomes at 30 days after positive cultures, patient characteristics, and drug prescription status. Patient background information included 
gender, previous hospitalization, stay in the intensive care unit (ICU), comorbidities, and history of surgery (within 90 days before candidemia onset) and drug exposure (administration for at least 2 days within 14 days before candidemia onset).

\subsection{Organism Identification and Susceptibility Testing}

Blood cultures were performed using the BacT/alert 3D system (bioMérieux, Lyon, France). All fungal isolates from blood cultures were identified with VITEK ${ }^{\mathrm{TM}_{2}}$ (bioMérieux, Lyon, France) using CHROMagar ${ }^{\mathrm{TM}}$ Candida broth (Becton Dickinson Japan, Tokyo, Japan).

The MIC measurement followed the methodology of the Clinical and Laboratory Standards Institute (CLSI) M27-A3 and used yeast-like fungi DP-Eiken (Tokyo, Japan), with higher values adopted when MICs differed in the same isolate. The MIC measurement ranges were as follows: fluconazole $0.12-64 \mu \mathrm{g} / \mathrm{mL}$, itraconazole and voriconazole $0.015-8 \mu \mathrm{g} / \mathrm{mL}$, amphotericin B and caspofungin $0.03-16 \mu \mathrm{g} / \mathrm{mL}$, and micafungin $0.015-16 \mu \mathrm{g} / \mathrm{mL}$. Posaconazole and anidulafungin were not approved in Japan during the study period. $\mathrm{MIC}_{50}$ and $\mathrm{MIC}_{90}$ were calculated for each species. $\mathrm{MIC}_{50}$ and $\mathrm{MIC}_{90}$ are defined as the concentrations of each antifungal agent necessary to inhibit $50 \%$ and $90 \%$ of the isolates, respectively. MIC $>0.06 \mu \mathrm{g} / \mathrm{mL}$ was used as a criterion for the low susceptibility of micafungin for non-parapsilosis Candida species. MIC $>0.06 \mu \mathrm{g} / \mathrm{mL}$ was set with reference to the resistance norm of C. glabrata in CLSI M60 1st Edition (Performance Standards for Antifungal Susceptibility Testing of Yeasts) [23].

\subsection{Antifungal Use}

Clinical departments were divided into four categories: hematology, internal medicine, surgery, and others, and antifungal use was calculated by dividing the days of therapy by 1000 patient days (PDs) [24].

\subsection{Factorial Analysis}

Multivariate analyses were performed on isolates with available patient backgrounds to determine factors that increased the risk of $C$. parapsilosis detection and those that resulted in low susceptibility to micafungin (MIC $>0.06 \mu \mathrm{g} / \mathrm{mL}$ ) among non-parapsilosis Candida species.

\subsection{Statistical Analysis}

Categorical variables were analyzed using Fisher's exact tests. Logistic regression analysis was applied to identify demographic and clinical variables associated with C. parapsilosis and with candidemia with MIC of micafugnin $>0.06 \mu \mathrm{g} / \mathrm{mL}$. Variables with a $p<0.20$ by bivariate analysis were included in multivariable model selection. Model selection was conducted using stepwise logistic regression and consideration of 2-way interaction terms. The level of significance was set at $\alpha=0.05$. All statistical analyses were performed using the statistical software package IBM-SPSS statistics 26.0 (IBM, New York, NY, USA).

\section{Results}

\subsection{Species Distribution of the Isolates and Mortality Rate}

The overall species distribution is shown in Table 1. During the study period, a total of 153 Candida clinical blood isolates were collected. C. albicans was the most prevalent species, followed by C. parapsilosis, C. glabrata, C. tropicalis, and C. famata. These five species accounted for more than $90 \%$ of all isolates. Twelve isolates were unidentified to the species level. Thirty-day mortality was $23.5 \%$ overall and especially more than $30 \%$ in C. tropicalis, C. famata, and C. krusei.

\subsection{Amount of Antifungal Usage and Species Distribution of Blood Isolated Candidemia from 2010 to 2018}

Antifungal use in days of therapy (DOT)/1000 patient days (PDs) by department was $673.0,15.2,20.2$, and 65.0 for hematology, internal medicine, surgery, and others, 
respectively (Table 2). When compared by the drug, the hematology department had the highest amount of antifungal use for azoles, echinocandins, and liposomal amphotericin B.

Table 1. Species distribution of Candida blood isolates.

\begin{tabular}{ccc}
\hline Species & No. (\%) of Isolates & 30-Day Mortality Rates \\
\hline Candida albicans & $59(38.6)$ & 23.7 \\
\hline Candida parapsilosis & $42(27.5)$ & 19.0 \\
\hline Candida glabrata & $21(13.7)$ & 23.8 \\
\hline Candida tropicalis & $11(7.2)$ & 36.4 \\
\hline Candida famata & $5(3.3)$ & 60.0 \\
\hline Candida krusei $^{\text {Candida lusitaniae }}$ & $2(1.3)$ & 50.0 \\
\hline Other $^{+}$ & $1(0.7)$ & 0.3 \\
\hline Total & $12(7.8)$ & 23.5 \\
\hline
\end{tabular}

${ }^{\dagger}$ Not identified to species further than the genus Candida.

Table 2. Antifungal use in days of therapy (DOT)/1000 patient days (PDs) among different hospital departments from 2010 to 2018 .

\begin{tabular}{|c|c|c|c|c|}
\hline & Hematology & Internal Medicine & Surgery & Others \\
\hline Micafungin & 181.9 & 4.3 & 10.7 & 9.2 \\
\hline Caspofungin & 5.9 & 0.1 & 0.2 & 0.2 \\
\hline Fluconazole & 104.1 & 5.1 & 7.1 & 42.7 \\
\hline Fosfluconazole & 0.3 & 0.1 & 0.1 & 0.0 \\
\hline Itraconazole & 332.6 & 2.0 & 0.3 & 4.1 \\
\hline Voriconazole & 38.8 & 2.6 & 0.9 & 6.6 \\
\hline $\begin{array}{c}\text { Liposomal } \\
\text { Amphotericin B }\end{array}$ & 9.3 & 0.6 & 1.1 & 2.0 \\
\hline Amphotericin B & 0.0 & 0.0 & 0.0 & 0.1 \\
\hline Flucytosine & 0.0 & 0.4 & 0.0 & 0.1 \\
\hline Total & 673.0 & 15.2 & 20.2 & 65.0 \\
\hline
\end{tabular}

The total does not account for patients who may have received multiple drugs on the same day.

In terms of species distribution by department, C. albicans was lower (9\%) and C. parapsilosis was higher $(41 \%)$ in the hematology department than in the other departments (Figure 1). The numbers of isolates detected during the survey were 22, 42, 71, and 18 for hematology, internal medicine, surgery, and others, respectively. For the number of isolates per 10,000 patient days (PDs), hematology was the highest (3.0), followed by surgery (1.4), internal medicine (0.84), and others (0.3) (Figure 1). In hematology, the rate of prophylaxis was $95.5 \%$.

\subsection{Factorial Analysis for C. parapsilosis Associated with Candidemia}

Univariate and multivariate analyses were performed on 147 isolates for factors that increased the risk of detecting C. parapsilosis (Table 3). Since detailed information was not available, six isolates were excluded in this analysis. Prior micafungin use increased the frequency of detecting C. parapsilosis and the multivariate analysis revealed that prior micafungin use was a risk factor for $C$. parapsilosis detection (odds ratio $(\mathrm{OR})=4.22 ; 95 \%$ confidence interval $(\mathrm{CI})=1.39-12.78 ; p=0.011$. ICU stay significantly decreased the frequency of detecting C. parapsilosis and multivariate analysis revealed that ICU stay was a risk factor for the onset 
of non-parapsilosis Candida species ( $\mathrm{OR}=0.276 ; 95 \% \mathrm{CI}=0.094-0.809 ; p=0.019)$. Chronic renal disease also significantly decreased the frequency of detecting $C$. parapsilosis; however, multivariate analysis revealed no significant differences $(\mathrm{OR}=0.441 ; 95 \% \mathrm{CI}=0.190-1.027$; $p=0.058$ ). Admission to the internal medicine department significantly decreased the frequency of detecting C. parapsilosis. However, patient department was not included in the multivariate analysis due to multicollinearity with micafungin exposure. There were no differences between the two groups regarding gender, history of hospitalization within 90 days, diabetes mellitus, organ transplantation, hematopoietic stem cell transplantation, neutropenia, gastrointestinal surgery, renal replacement therapy, and the administration of antimicrobials, steroids, and immunosuppressants.

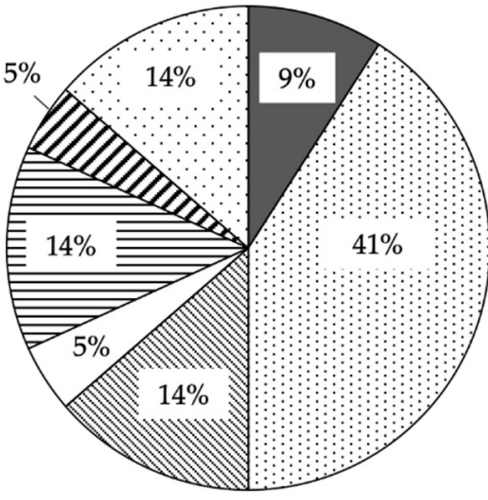

Hematology (3.0 isolates/10,000 PDs)

\section{Candida albicans}

目 Candida famata

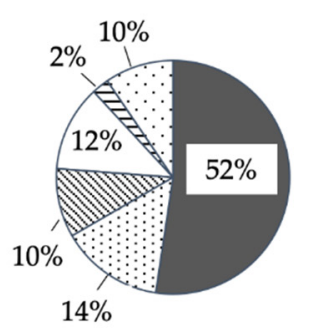

Internal medicine (0.8 isolates/10,000 PDs)

\section{Candida parapsilosis}

Z Candida krusei
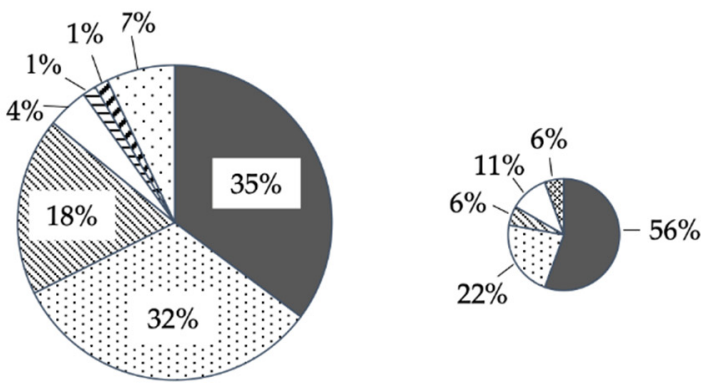

Surgery

(1.4 isolates/10,000 PDs) (0.2 isolates/10,000 PDs)

Sandida glabrata

图 Candida lusitaniae

\section{$\square$ Candida tropicalis}

Other Candida spp.

Figure 1. Species distribution of Candida blood isolates among different hospital departments from 2010 to 2018 . Species distribution by clinical department is shown. The area ratio of the pie chart shows the number of cases per 10,000 patient days (PDs). The rate number is shown in the figure. Note: The total is not $100 \%$ because the data are rounded to integers.

\subsection{Antifungal MIC Distribution of Candida Blood Isolates}

The $\mathrm{MIC}_{90}$ of caspofungin for Candida spp. was $0.5-4 \mu \mathrm{g} / \mathrm{mL}$ and the $\mathrm{MIC}_{90}$ of micafungin was $0.03-1 \mu \mathrm{g} / \mathrm{mL}$ (Table 4 ). The $\mathrm{MIC}_{50}$ of caspofungin and micafungin for C. parapsilosis was $1 \mu \mathrm{g} / \mathrm{mL}$ and $0.5 \mu \mathrm{g} / \mathrm{mL}$, respectively, and the $\mathrm{MIC}_{90}$ of both was $1 \mu \mathrm{g} / \mathrm{mL}$. Apart from C. parapsilosis, C. glabrata showed higher MICs compared to the other species. The $\mathrm{MIC}_{50}$ of caspofungin and micafungin for C. glabrata was $1 \mu \mathrm{g} / \mathrm{mL}$ and $0.03 \mu \mathrm{g} / \mathrm{mL}$, whereas the $\mathrm{MIC}_{90}$ was $4 \mu \mathrm{g} / \mathrm{mL}$ and $1 \mu \mathrm{g} / \mathrm{mL}$, respectively.

Table 3. Risk factors for bloodstream infections caused by Candida parapsilosis. (bivariate and multivariate analyses).

\begin{tabular}{|c|c|c|c|c|c|c|c|}
\hline Factor & $\begin{array}{c}\text { Non-parapsilosis } \\
\text { Candida Species } \\
(n=108)\end{array}$ & $\begin{array}{l}\text { C. parapsilosis } \\
\quad(n=39)\end{array}$ & $p$ & $\begin{array}{c}\text { Unadjusted } \\
\text { OR } \\
(95 \% \mathrm{CI})\end{array}$ & $p$ & $\begin{array}{l}\text { Adjusted OR } \\
\text { (95\% CI) }\end{array}$ & $p$ \\
\hline No. (\%) male patients & $69(64)$ & $27(69)$ & 0.695 & & & & \\
\hline $\begin{array}{l}\text { Hospitalization in } \\
\text { prior } 90 \text { days }\end{array}$ & $56(52)$ & $16(55)$ & 0.267 & & & & \\
\hline ICU stay & $38(35)$ & $6(15)$ & 0.025 & $\begin{array}{c}0.307 \\
(0.102-0.923)\end{array}$ & 0.036 & $\begin{array}{c}0.276 \\
(0.094-0.809)\end{array}$ & 0.019 \\
\hline \multicolumn{8}{|c|}{ No. (\%) of patient department: } \\
\hline Hematology & $13(12)$ & $7(18)$ & 0.415 & & & & \\
\hline
\end{tabular}


Table 3. Cont.

\begin{tabular}{|c|c|c|c|c|c|c|c|}
\hline Factor & $\begin{array}{l}\text { Non-parapsilosis } \\
\text { Candida Species } \\
(n=108)\end{array}$ & $\begin{array}{l}\text { C. parapsilosis } \\
(n=39)\end{array}$ & $p$ & $\begin{array}{c}\text { Unadjusted } \\
\text { OR } \\
(95 \% \mathrm{CI})\end{array}$ & $p$ & $\begin{array}{l}\text { Adjusted OR } \\
\text { (95\% CI) }\end{array}$ & $p$ \\
\hline Internal medicine & $35(32)$ & $5(13)$ & 0.021 & & & & \\
\hline Surgery & $46(43)$ & $23(56)$ & 0.094 & & & & \\
\hline Others & $14(13)$ & $4(10)$ & 0.781 & & & & \\
\hline \multicolumn{8}{|c|}{ No. (\%) of patients with: } \\
\hline Diabetes & $23(21)$ & $6(15)$ & 0.490 & & & & \\
\hline Chronic renal disease & $54(50)$ & $12(31)$ & 0.041 & $\begin{array}{c}0.443 \\
(0.187-1.05) \\
\end{array}$ & 0.063 & $\begin{array}{c}0.441 \\
(0.190-1.027) \\
\end{array}$ & 0.058 \\
\hline HIV infection & $0(0)$ & $0(0)$ & - & & & & \\
\hline $\begin{array}{l}\text { Solid organ } \\
\text { transplantation }\end{array}$ & $2(2)$ & $0(0)$ & 1.00 & & & & \\
\hline $\begin{array}{l}\text { Bone marrow } \\
\text { transplantation }\end{array}$ & $4(4)$ & $4(10)$ & 0.209 & & & & \\
\hline $\begin{array}{l}\text { Neutropenia } \\
(<500 / \mu \mathrm{L})\end{array}$ & $9(8)$ & $4(10)$ & 0.746 & & & & \\
\hline \multicolumn{8}{|c|}{ No. (\%) of patients who underwent invasive procedures } \\
\hline $\begin{array}{l}\text { Gastrointestinal } \\
\text { surgery }\end{array}$ & $13(12)$ & $5(13)$ & 1.00 & & & & \\
\hline $\begin{array}{l}\text { Renal replacement } \\
\text { therapy }\end{array}$ & $17(16)$ & $3(8)$ & 0.281 & & & & \\
\hline Tunneled catheter & $3(3)$ & $4(10)$ & 0.081 & $\begin{array}{c}2.17 \\
(0.383-12.2) \\
\end{array}$ & 0.382 & & \\
\hline $\begin{array}{l}\text { Nontunneled } \\
\text { catheter }\end{array}$ & $84(78)$ & $26(67)$ & 0.198 & $\begin{array}{c}0.6 \\
(0.228-1.58) \\
\end{array}$ & 0.302 & & \\
\hline \multicolumn{8}{|c|}{ No. $(\%)$ of patients with previous: } \\
\hline Antibiotic treatment & $78(72)$ & $24(62)$ & 0.229 & & & & \\
\hline Fluconazole exposure & $2(2)$ & $0(0)$ & 1.00 & & & & \\
\hline $\begin{array}{l}\text { Itraconazole } \\
\text { exposure }\end{array}$ & $2(2)$ & $1(3)$ & 1.00 & & & & \\
\hline $\begin{array}{l}\text { Voriconazole } \\
\text { exposure }\end{array}$ & $1(1)$ & $0(0)$ & 1.00 & & & & \\
\hline Micafungin exposure & $13(12)$ & $9(23)$ & 0.118 & $\begin{array}{c}4.97 \\
(1.56-15.5) \\
\end{array}$ & 0.006 & $\begin{array}{c}4.22 \\
(1.39-12.8) \\
\end{array}$ & 0.011 \\
\hline $\begin{array}{l}\text { Liposomal } \\
\text { Amphotericin B } \\
\text { exposure }\end{array}$ & $1(1)$ & $0(0)$ & 1.00 & & & & \\
\hline $\begin{array}{c}\text { Corticosteroid } \\
\text { treatment }\end{array}$ & $29(27)$ & $11(28)$ & 1.00 & & & & \\
\hline $\begin{array}{l}\text { Immunosuppression } \\
\text { medications }\end{array}$ & $11(10)$ & $6(15)$ & 0.391 & & & & \\
\hline
\end{tabular}

Abbreviations: CI, confidence interval; ICU, intensive care unit; HIV, human immunodeficiency virus. Bivariate analyses were analyzed using Fisher's exact tests. Variables with a $p<0.20$ by bivariate analysis were included in multivariable model selection. Model selection was conducted using stepwise logistic regression and consideration of 2-way interaction terms. The level of significance was set at $\alpha=0.05$. 
Table 4. Minimum inhibitory concentrations (MICs) of Candida blood isolates after $24 \mathrm{~h}$ of incubation.

\begin{tabular}{|c|c|c|c|c|c|c|c|c|c|c|c|c|c|c|c|c|c|}
\hline \multirow{2}{*}{\multicolumn{2}{|c|}{ Species and Antifungal Agent (No. of Isolates) }} & \multicolumn{14}{|c|}{ No. of Isolates with MIC (mg/mL) of: } & \multirow{2}{*}{$\underset{\left(\mu g_{5 / m L}\right)}{\operatorname{MIC}_{50}}$} & \multirow{2}{*}{$\underset{(\mu \mathrm{g} / \mathrm{mL}}{\mathrm{MIC}_{90}}$} \\
\hline & & 0.015 & 0.03 & 0.06 & 0.12 & 0.25 & 0.5 & 1 & 2 & 4 & 8 & 16 & 32 & 64 & $>64$ & & \\
\hline C. albicans (57) & $\begin{array}{c}\text { Fluconazole } \\
\text { Itraconazole } \\
\text { Voriconazole } \\
\text { Amphotericin B } B \\
\text { Caspofungin } ₹ \\
\text { Micafungin } \\
\end{array}$ & $\begin{array}{r}5 \\
35 \\
\\
\\
\end{array}$ & $\begin{array}{c}14 \\
10 \\
1 \\
7 \\
\end{array}$ & $\begin{array}{l}21 \\
6 \\
1\end{array}$ & $\begin{array}{c}19 \\
11 \\
2 \\
4 \\
2\end{array}$ & $\begin{array}{c}18 \\
2 \\
1 \\
24 \\
12 \\
1 \\
\end{array}$ & $\begin{array}{c}6 \\
3 \\
2 \\
22 \\
3\end{array}$ & 4 & 1 & 1 & & $\begin{array}{l}1^{+} \\
1^{+}\end{array}$ & & 1 & 1 & $\begin{array}{c}0.25 \\
0.06 \\
\leq 0.01 \\
0.25 \\
0.25 \\
\leq 0.01 \\
\end{array}$ & $\begin{array}{c}2 \\
0.25 \\
0.12 \\
0.5 \\
0.5 \\
0.03 \\
\end{array}$ \\
\hline C. parapsilosis (39) & $\begin{array}{l}\text { Fluconazole } \\
\text { IItraconazale } \\
\text { Voriconazole } \\
\text { Amphotericin B } \\
\text { Caspofungin } ₹ \\
\text { Micafungin } \\
\end{array}$ & 23 & $\begin{array}{c}3 \\
15\end{array}$ & 14 & $\begin{array}{c}1 \\
19 \\
1 \\
6 \\
1 \\
1\end{array}$ & $\begin{array}{c}5 \\
2 \\
13 \\
4 \\
\end{array}$ & $\begin{array}{c}20 \\
1 \\
18 \\
2 \\
23 \\
\end{array}$ & $\begin{array}{c}2 \\
7 \\
10 \\
\end{array}$ & & & & 1 & & & & $\begin{array}{c}0.5 \\
0.12 \\
0.015 \\
0.5 \\
1 \\
0.5 \\
\end{array}$ & $\begin{array}{c}1 \\
0.12 \\
0.03 \\
0.5 \\
1 \\
1 \\
\end{array}$ \\
\hline C. glabaratata(20) & $\begin{array}{c}\text { Fluconazole } \\
\text { Itraconazale } \\
\text { Voriconazole } \\
\text { Amphotericin } B \\
\text { Caspofungin } \neq \\
\text { Micafungin }\end{array}$ & 3 & 1 & $\begin{array}{l}1 \\
4\end{array}$ & $\begin{array}{l}3 \\
5 \\
1 \\
1 \\
\end{array}$ & $\begin{array}{l}6 \\
6 \\
5 \\
2 \\
2\end{array}$ & $\begin{array}{c}1 \\
6 \\
1 \\
11 \\
3 \\
2 \\
2\end{array}$ & $\begin{array}{l}4 \\
4 \\
3 \\
3 \\
3 \\
\end{array}$ & & 7 & 6 & 2 & & & & $\begin{array}{c}4 \\
0.25 \\
0.12 \\
0.5 \\
1 \\
0.03 \\
\end{array}$ & $\begin{array}{c}16 \\
1 \\
0.25 \\
1 \\
4 \\
1 \\
\end{array}$ \\
\hline C. Iropicalis (11) & $\begin{array}{l}\text { Fluconazole } \\
\text { Itraconazole } \\
\text { Voriconazole } \\
\text { Amphotericin B } \\
\text { Caspofungin } ₹ \\
\text { Micafungin }\end{array}$ & 2 & $\begin{array}{l}1 \\
4 \\
\\
7\end{array}$ & 5 & $\begin{array}{l}1 \\
1\end{array}$ & $\begin{array}{l}4 \\
1 \\
1 \\
3 \\
2\end{array}$ & $\begin{array}{l}2 \\
1 \\
8 \\
5\end{array}$ & $\begin{array}{l}1 \\
1\end{array}$ & $\begin{array}{l}1 \\
1\end{array}$ & 1 & & $\begin{array}{l}1^{\dagger} \\
1^{+}\end{array}$ & & 1 & 2 & $\begin{array}{c}0.5 \\
0.06 \\
0.03 \\
0.5 \\
0.5 \\
0.03 \\
\end{array}$ & $\begin{array}{c}>64 \\
2 \\
4 \\
0.5 \\
0.5 \\
0.03 \\
\end{array}$ \\
\hline C. famata (5) & $\begin{array}{l}\text { Fluconazole } \\
\text { IIraconazole } \\
\text { Voriconazole } \\
\text { Amphotericin B } \\
\text { Caspofungin } ₹ \\
\text { Micafungin } \\
\end{array}$ & 1 & 1 & ${ }_{3}^{1}$ & 2 & $\begin{array}{l}4 \\
3 \\
2 \\
2 \\
\end{array}$ & $\begin{array}{l}2 \\
1 \\
1 \\
\end{array}$ & 2 & 3 & & & & & & & $\begin{array}{c}2 \\
0.25 \\
0.06 \\
0.25 \\
0.5 \\
0.25 \\
\end{array}$ & $\begin{array}{c}2 \\
0.25 \\
0.06 \\
0.5 \\
1 \\
0.5 \\
\end{array}$ \\
\hline C. krissel (2) & $\begin{array}{c}\text { Fluconazole \& } \\
\text { Itraconazole } \\
\text { Voriconazole } \\
\text { Amphotericin B } \\
\text { Caspofungin } ₹ \\
\text { Micafungin } \\
\end{array}$ & & 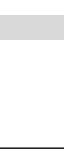 & 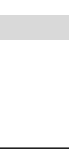 & 2 & $\begin{array}{l}2 \\
2\end{array}$ & 1 & $\begin{array}{l}1 \\
2\end{array}$ & & & & 1 & 1 & & & $\begin{array}{c}16 \\
0.25 \\
0.25 \\
0.5 \\
1 \\
0.12 \\
\end{array}$ & $\begin{array}{c}32 \\
0.25 \\
0.25 \\
1 \\
1 \\
0.12 \\
\end{array}$ \\
\hline Uner andul SPP. (18) & $\begin{array}{l}\text { Fluconazole } \\
\text { Itraconazole } \\
\text { Voriconazole } \\
\text { Amphotericin B } \\
\text { Caspofungin } \neq \\
\text { Micafungin }\end{array}$ & 6 & 1 & $\begin{array}{l}4 \\
4\end{array}$ & $\begin{array}{l}4 \\
1 \\
1 \\
3 \\
\end{array}$ & $\begin{array}{l}3 \\
4 \\
1 \\
8 \\
\\
3 \\
\end{array}$ & $\begin{array}{l}4 \\
1 \\
3 \\
3 \\
4 \\
\end{array}$ & $\begin{array}{l}1 \\
5 \\
2 \\
\end{array}$ & 3 & 2 & & & +5 & & & $\begin{array}{c}0.5 \\
0.12 \\
0.03 \\
0.25 \\
1 \\
0.25 \\
\end{array}$ & $\begin{array}{c}4 \\
0.25 \\
0.12 \\
0.5 \\
1 \\
1 \\
\end{array}$ \\
\hline
\end{tabular}

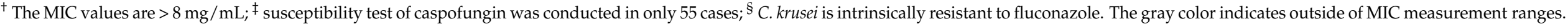




\subsection{Factorial Analysis for Low Micafungin Susceptibility in Non-parapsilosis Candida Species}

Among non-parapsilosis Candida species, we performed univariate and multivariate analyses in 108 isolates to determine the factors leading to decreased susceptibility of micafungin (MIC $>0.06 \mu \mathrm{g} / \mathrm{mL}$ ). In hematology (MIC $\leq 0.06 \mu \mathrm{g} / \mathrm{mL}: 5.0 \%$ vs $\mathrm{MIC}>0.06 \mu \mathrm{g} / \mathrm{mL}$ : $32.1 \%$; $p<0.001$ ), neutropenia (MIC $\leq 0.06 \mu \mathrm{g} / \mathrm{mL}: 3.8 \%$ vs $\mathrm{MIC}>0.06 \mu \mathrm{g} / \mathrm{mL}: 21.4 \%$; $p=0.009$ ), CV catheterization (MIC $\leq 0.06 \mu \mathrm{g} / \mathrm{mL}: 72.5 \%$ vs $\mathrm{MIC}>0.06 \mu \mathrm{g} / \mathrm{mL}: 92.9 \%$; $p=0.033$ ), prior micafungin use (MIC $\leq 0.06 \mu \mathrm{g} / \mathrm{mL}: 3.8 \%$ vs MIC $>0.06 \mu \mathrm{g} / \mathrm{mL}: 35.7 \%$; $p<0.001$ ), and immunosuppressant use (MIC $\leq 0.06 \mu \mathrm{g} / \mathrm{mL}: 6.3 \%$ vs $\mathrm{MIC}>0.06 \mu \mathrm{g} / \mathrm{mL}$ : $21.4 \% ; p=0.033$ ) the detection frequency was significantly higher in the MIC $>0.06 \mu \mathrm{g} / \mathrm{mL}$ isolates (Table 5). Multivariate analysis revealed that prior micafungin use was a significant risk factor for an increased frequency of detecting isolates with MIC $>0.06 \mu \mathrm{g} / \mathrm{mL}$ $(\mathrm{OR}=13.24 ; 95 \% \mathrm{CI}=3.23-54.2 ; p<0.01)$. Patient department was not included in the multivariate analysis due to multicollinearity with micafungin exposure. There were no differences regarding gender, a history of hospital stay within 90 days, ICU stay, diabetes mellitus, organ transplantation, hematopoietic stem cell transplantation, gastrointestinal surgery, renal replacement therapy, CV port and administration of antibiotics or steroids between the two groups.

Table 5. Risk factors for bloodstream infections caused by non-parapsilosis Candida species with a minimum inhibitory concentration (MIC) of micafungin $>0.06 \mu \mathrm{g} / \mathrm{mL}$. (Bivariate and multivariate analyses.)

\begin{tabular}{|c|c|c|c|c|c|c|c|}
\hline Factor & $\begin{array}{c}\mathrm{MIC} \leq 0.06 \\
\mu \mathrm{g} / \mathrm{mL} \\
(n=80)\end{array}$ & $\begin{array}{c}\mathrm{MIC}>0.06 \\
\mu \mathrm{g} / \mathrm{mL} \\
(n=28)\end{array}$ & $p$ & $\begin{array}{c}\text { Unadjusted } \\
\text { OR } \\
(95 \% \mathrm{CI})\end{array}$ & $p$ & $\begin{array}{c}\text { Adjusted } \\
\text { OR } \\
(95 \% \text { CI })\end{array}$ & $p$ \\
\hline No. $(\%)$ male patients & $51(64)$ & $18(64)$ & 1.00 & & & & \\
\hline $\begin{array}{l}\text { Hospitalization in prior } \\
90 \text { days }\end{array}$ & $38(48)$ & $16(57)$ & 0.511 & & & & \\
\hline ICU stay & $26(33)$ & $12(43)$ & 0.362 & & & & \\
\hline \multicolumn{8}{|c|}{ No. (\%) of patient department: } \\
\hline Hematology & $4(5)$ & $9(32)$ & $<0.001$ & & & & \\
\hline Internal medicine & $30(38)$ & $5(18)$ & 0.064 & & & & \\
\hline Surgery & $33(41)$ & $13(46)$ & 0.662 & & & & \\
\hline Others & $13(16)$ & $1(4)$ & 0.109 & & & & \\
\hline \multicolumn{8}{|l|}{ No. (\%) of patients with: } \\
\hline Diabetes & $15(19)$ & $8(29)$ & 0.292 & & & & \\
\hline Chronic renal disease & $39(49)$ & $15(54)$ & 0.669 & & & & \\
\hline HIV infection & $0(0)$ & $0(0)$ & - & & & & \\
\hline $\begin{array}{c}\text { Solid organ } \\
\text { transplantation }\end{array}$ & $1(1)$ & $1(4)$ & 0.453 & & & & \\
\hline $\begin{array}{l}\text { Bone marrow } \\
\text { transplantation }\end{array}$ & $2(3)$ & $2(7)$ & 0.276 & & & & \\
\hline Neutropenia $(<500 / \mu \mathrm{L})$ & $3(4)$ & $6(21)$ & 0.009 & $\begin{array}{c}0.762 \\
(0.094-6.18)\end{array}$ & 0.799 & & \\
\hline \multicolumn{8}{|c|}{ No. (\%) of patients who underwent invasive procedures } \\
\hline Gastrointestinal surgery & $11(14)$ & $2(7)$ & 0.508 & & & & \\
\hline $\begin{array}{c}\text { Renal replacement } \\
\text { therapy }\end{array}$ & $12(15)$ & $5(18)$ & 0.766 & & & & \\
\hline Tunneled catheter & $3(4)$ & $0(0)$ & 0.567 & & & & \\
\hline Nontunneled catheter & $58(73)$ & $26(93)$ & 0.033 & $\begin{array}{c}4.61 \\
(0.846-25.1)\end{array}$ & 0.077 & & \\
\hline
\end{tabular}


Table 5. Cont

\begin{tabular}{|c|c|c|c|c|c|c|c|}
\hline Factor & $\begin{array}{c}\mathrm{MIC} \leq 0.06 \\
\mu \mathrm{g} / \mathrm{mL} \\
(n=80)\end{array}$ & $\begin{array}{c}\mathrm{MIC}>0.06 \\
\mu \mathrm{g} / \mathrm{mL} \\
(n=28)\end{array}$ & $p$ & $\begin{array}{c}\text { Unadjusted } \\
\text { OR } \\
(95 \% \mathrm{CI})\end{array}$ & $p$ & $\begin{array}{c}\text { Adjusted } \\
\text { OR } \\
(95 \% \text { CI })\end{array}$ & $p$ \\
\hline \multicolumn{8}{|c|}{ No. (\%) of patients with previous: } \\
\hline Antibiotic treatment & $55(69)$ & $23(82)$ & 0.224 & & & & \\
\hline Micafungin exposure & $3(4)$ & $10(36)$ & $<0.001$ & $\begin{array}{c}11.5 \\
(1.91-69.1) \\
\end{array}$ & 0.008 & $\begin{array}{c}13.2 \\
(3.23-54.2) \\
\end{array}$ & $<0.01$ \\
\hline Corticosteroid treatment & $20(25)$ & $9(32)$ & 0.467 & & & & \\
\hline $\begin{array}{l}\text { Immunosuppression } \\
\text { medications }\end{array}$ & $5(6)$ & $6(21)$ & 0.032 & $\begin{array}{c}5.44 \\
(1.05-28.1)\end{array}$ & 0.043 & $\begin{array}{c}3.44 \\
(0.831-14.2)\end{array}$ & 0.088 \\
\hline
\end{tabular}

Abbreviations: CI, confidence interval; ICU, intensive care unit; HIV, human immunodeficiency Virus. Bivariate analyses were analyzed using Fisher's exact tests. Variables with a $p<0.20$ by bivariate analysis were included in multivariable model selection. Model selection was conducted using stepwise logistic regression and consideration of 2-way interaction terms. The level of significance was set at $\alpha=0.05$.

\section{Discussion}

The percentage of detected species (Table 1) was similar with previous studies from Japan [17-19], while it differed from the results from the United States. In the USA hospitalbased antifungal use survey [25], echinocandin antifungal use accounted for $14 \%$ of all antifungals, whereas in the present study, echinocandin use was $25.1 \%$. This difference in antifungal use affected the detected fungal species. The present study used mainly micafungin among echinocandins and the frequency of $C$. parapsilosis occurrence was elevated, which is similar with the study predominantly used caspofungin [21]. For the first time to date, we showed that increasing micafungin use increased the frequency of C. parapsilosis occurrence. While prior administration of fluconazole has been reported to be a risk factor for the breakthrough of C. glabrata and C. krusei [26], we showed for the first time that prior micafungin use is a risk factor for the breakthrough of $C$. parapsilosis.

In the antifungal use by department, the use of antifungal drugs was the highest in the hematology department. This department also had the highest detection frequency of non-albicans Candida species such as C. parapsilosis. Patients with hematological malignancy use more antifungal agents because antifungal prophylaxis is recommended in many guidelines $[12,14,27-29]$. Patients with hematological malignancy have a higher risk and frequency of developing deep mycoses. Fluconazole, itraconazole, voriconazole, and micafungin are recommended as prophylactic agents in the Japanese guidelines $[12,14]$. However, fluconazole is not active against Aspergillus spp. and itraconazole can often not be continued due to gastrointestinal toxicity [30] despite improved absorption with oral solutions. In CYP2C19 poor metabolizers, blood levels of voriconazole are likely to increase, with the percentage of poor metabolizers higher in Japanese than in Western individuals. Therefore, Japanese patients develop hepatic dysfunction more frequently due to voriconazole [31]. For these reasons, micafungin is often chosen for prophylaxis in Japan. Arendrup et al. reported an increase of non-albicans Candida species when the duration of antifungal use was at least 7 days prior to the detection of culture outcome [1,32]. In our study, prophylaxis was provided in $95.5 \%$ of hematology department isolates. The higher use of antifungals, including prophylaxis, was the reason for the higher rate of detecting non-albicans Candida species. In addition, the present study revealed that prior micafungin use is a risk factor for low susceptibility (Table 5). Compared with the previous study result, $\mathrm{MIC}_{90}$ of $C$. glabrata was $0.06-0.25 \mu \mathrm{g} / \mathrm{mL}$ in a Japanese study $[17,19]$; therefore, our results showed $\mathrm{MIC}_{90}$ for $\mathrm{C}$. glabrata increased. The same as breakthrough infections with higher MICs occurring during micafungin use in C. glabrata [33], we showed prior micafungin use not only increased the frequency of $C$. parapsilosis but also is associated with low susceptibility in non-parapsilosis Candida species. The criteria for susceptibility remain controversial; therefore, in the present study, low susceptibility was defined as MIC $>0.06 \mu \mathrm{g} / \mathrm{mL}$ with reference to the criteria for resistance of C. glabrata in CLSI 
M60 1st Edition [23]. Andes et al. has reported an MIC of $0.06 \mu \mathrm{g} / \mathrm{mL}$ for $\geq 90 \%$ therapeutic efficacy at micafungin $100 \mathrm{mg} /$ day administration considering the pharmacokinetics / pharmacodynamics (PK/PD) parameters [34]; as such, we considered it reasonable to define low susceptibility as MIC $>0.06 \mu \mathrm{g} / \mathrm{mL}$.

Our results showed that the overall 30 -day mortality rate was $23.5 \%$, which was lower than other studies on overall mortality (39.3\%) of nosocomial bloodstream infections caused by Candida in Japan [2]. Candidemia caused by C. parapsilosis is associated with a lower mortality rate $[35,36]$, which could be attributed to the higher frequency of $C$. parapsilosis occurrence in our study. In contrast, the mortality rates of candidemia caused by $C$. tropicalis, C. famata, and C. krusei were as high as $36.4 \%, 50.0 \%$, and $60.0 \%$, respectively. Previous studies also showed that $C$. tropicalis and $C$. krusei candidemia had mortality rates of $43.1 \%$ and $58.7 \%$ [3], indicating similar results.

The present study had several limitations. First, it was a single-center retrospective study. A multicenter observational study [18] was conducted in Japan; however, it did not investigate whether antifungal agents affected the detected species or their susceptibilities. The results of our multivariate analyses might be influenced by the sample size and number of variables included in the models. In the future, similar considerations should be made in multiple centers with differing antifungal use status. Second, low susceptibility was defined as MIC $>0.06 \mu \mathrm{g} / \mathrm{mL}$ in the present study. The possibility of differing PK/PD parameters for each species has also been reported [37,38]; as such, MIC $>0.06 \mu \mathrm{g} / \mathrm{mL}$ is not the same as resistance. Third, fungal isolates were identified with only VITEKTM2 using the CHROMagarTM Candida broth. There are several reports of misidentification especially when C. famata is reported [39-42], so the possibility of misidentification must be considered. Although there are limitations, this is the first informative study to show that prior micafungin use affects the detected species and their respective MICs.

\section{Conclusions}

Prior micafungin use increased the risk of $C$. parapsilosis and the MIC $>0.06 \mu \mathrm{g} / \mathrm{mL}$ of micafungin in non-parapsilosis Candida species. Since there are only a few antifungal options for treatment, further antifungal stewardship considering azole-based antifungal use is required.

Author Contributions: Conceptualization, Y.S. (Yasutaka Sakamoto), K.K., T.S., K.S., K.I., T.N., Y.E., K.T., H.K. (Hirofumi Koike), H.K. (Hideaki Kato), Y.S. (Yukiko Sahashi), and K.M.; Data curation, Y.S. (Yasutaka Sakamoto), T.S., T.N., Y.E., K.T., and K.M.; Formal analysis, Y.S. (Yasutaka Sakamoto), Y.E., K.T., H.K. (Hideaki Kato), and K.M.; Investigation, Y.S. (Yasutaka Sakamoto), K.K., T.S., K.I., and T.N.; Methodology, Y.S. (Yasutaka Sakamoto), Y.E., K.T., H.K. (Hideaki Kato), and K.M.; Project administration, Y.E., K.T., H.K. (Hirofumi Koike), H.K. (Hideaki Kato), Y.S. (Yukiko Sahashi), and K.M.; Resources, K.S. and H.K. (Hideaki Kato); Software, Y.S. (Yasutaka Sakamoto), Y.E., K.T., Y.S. (Yukiko Sahashi), and K.M.; Supervision, Y.E., K.T., H.K. (Hirofumi Koike), H.K. (Hideaki Kato), Y.S. (Yukiko Sahashi ), and K.M.; Validation, Y.S. (Yasutaka Sakamoto), K.K., T.S., K.S., K.I., T.N., Y.E., K.T., H.K. (Hirofumi Koike), Y.S. (Yukiko Sahashi), and K.M.; Visualization, Y.S. (Yasutaka Sakamoto), K.K., T.S., K.S., K.I., T.N., Y.E., K.T., H.K. (Hirofumi Koike), H.K. (Hideaki Kato), Y.S. (Yukiko Sahashi), and K.M.; Writing—original draft, Y.S. (Yasutaka Sakamoto), K.K., T.S., K.S., K.I., T.N., Y.E., K.T., H.K. (Hirofumi Koike), Y.S. (Yukiko Sahashi), and K.M.; Writing-review and editing, Y.S. (Yasutaka Sakamoto), K.K., T.S., K.S., K.I., T.N., Y.E., K.T., H.K. (Hirofumi Koike), H.K. (Hideaki Kato), Y.S. (Yukiko Sahashi), and K.M. All authors have read and agreed to the published version of the manuscript.

Funding: This research received no external funding.

Institutional Review Board Statement: The study was conducted according to the guidelines of the Declaration of Helsinki, and approved by the Yokohama City University Ethics Committee (protocol code B190600046 and date of approval 8 August 2019).

Informed Consent Statement: Informed consent was obtained from all subjects involved in the study. 
Data Availability Statement: The data presented in this study are available on request from the corresponding author. The data are not publicly available due to privacy.

Conflicts of Interest: The authors declare no conflict of interest.

\section{References}

1. Kullberg, B.J.; Arendrup, M.C. Invasive Candidiasis. N. Engl. J. Med. 2015, 373, 1445-1456. [CrossRef]

2. Nagao, M. A multicentre analysis of epidemiology of the nosocomial bloodstream infections in Japanese university hospitals. Clin. Microbiol. Infect. 2013, 19, 852-858. [CrossRef]

3. Wisplinghoff, H.; Bischoff, T.; Tallent, S.M.; Seifert, H.; Wenzel, R.P.; Edmond, M.B. Nosocomial Bloodstream Infections in US Hospitals: Analysis of 24,179 Cases from a Prospective Nationwide Surveillance Study. Clin. Infect. Dis. 2004, 39, 309-317. [CrossRef]

4. Gudlaugsson, O.; Gillespie, S.; Lee, K.; Berg, J.V.; Hu, J.; Messer, S.; Herwaldt, L.; Pfaller, M.; Diekema, D. Attributable Mortality of Nosocomial Candidemia, Revisited. Clin. Infect. Dis. 2003, 37, 1172-1177. [CrossRef]

5. Klingspor, L.; Tortorano, A.M.; Pemán, J.; Willinger, B.; Hamal, P.; Sendid, B.; Velegraki, A.; Kibbler, C.; Meis, J.; Sabino, R.F.P.; et al. Invasive Candida infections in surgical patients in intensive care units: A prospective, multicentre survey initiated by the European Confederation of Medical Mycology (ECMM) (2006-2008). Clin. Microbiol. Infect. 2015, 21, 87.e1-87.e10. [CrossRef]

6. Morgan, J.; Meltzer, M.I.; Plikaytis, B.D.; Sofair, A.N.; Huie-White, S.; Wilcox, S.; Harrison, L.H.; Seaberg, E.C.; Hajjeh, R.A.; Teutsch, S.M. Excess Mortality, Hospital Stay, and Cost Due to Candidemia: A Case-Control Study Using Data From PopulationBased Candidemia Surveillance. Infect. Control. Hosp. Epidemiol. 2005, 26, 540-547. [CrossRef]

7. Garey, K.W.; Rege, M.; Pai, M.P.; Mingo, D.E.; Suda, K.J.; Turpin, R.S.; Bearden, D. Time to Initiation of Fluconazole Therapy Impacts Mortality in Patients with Candidemia: A Multi-Institutional Study. Clin. Infect. Dis. 2006, 43, 25-31. [CrossRef]

8. Ben-Abraham, R. Predictors of adverse outcome from candidal infection in a tertiary care hospital. J. Infect. 2004, 49, 317-323. [CrossRef]

9. Pappas, P.G.; Kauffman, C.A.; Andes, D.; Clancy, C.J.; Marr, K.A.; Ostrosky-Zeichner, L.; Reboli, A.C.; Schuster, M.G.; Vazquez, J.A.; Walsh, T.J.; et al. Clinical Practice Guideline for the Management of Candidiasis: 2016 Update by the Infectious Diseases Society of America. Clin. Infect. Dis. 2016, 62, e1-e50. [CrossRef]

10. Cornely, O.A.; Bassetti, M.; Calandra, T.; Garbino, J.; Kullberg, B.; Lortholary, O.; Meersseman, W.; Akova, M.; Arendrup, M.; Arikan-Akdagli, S.; et al. ESCMID* guideline for the diagnosis and management of Candida diseases 2012: Non-neutropenic adult patients. Clin. Microbiol. Infect. 2012, 18, 19-37. [CrossRef] [PubMed]

11. Ullmann, A.; Akova, M.; Herbrecht, R.; Viscoli, C.; Arendrup, M.C.; Arikan-Akdagli, S.; Bassetti, M.; Bille, J.; Calandra, T.; Castagnola, E.; et al. ESCMID* Guideline for the diagnosis and management of Candida diseases 2012: Adults with haematological malignancies and after haematopoietic stem cell transplantation (HCT). Clin. Microbiol. Infect. 2012, 18, 53-67. [CrossRef]

12. Kohno, S.; Tamura, K.; Niki, Y.; Izumikawa, K.; Oka, S.; Ogawa, K.; Kadota, J.; Kamei, K.; Kanda, Y.; Kiuchi, T.; et al. Executive Summary of Japanese Domestic Guidelines for Management of Deep-seated Mycosis Med. Mycol. J. 2016, 57, E117-E163. [CrossRef]

13. Tashiro, S.; Osa, S.; Igarashi, Y.; Watabe, Y.; Liu, X.; Enoki, Y.; Taguchi, K.; Mayumi, T.; Miyazaki, Y.; Takesue, Y.; et al. Echinocandins versus non-echinocandins for the treatment of invasive candidiasis: A meta-analysis of randomized controlled trials. J. Infect. Chemother. 2020, 26, 1164-1176. [CrossRef] [PubMed]

14. Japan Society for Hematopoietic Cell Transplantation (JSHCT). Guideline for Prophylaxis and Treatment of Fungal Infection; JSHCT Monograph Volume; September 2017; Available online: https://www.jshct.com/uploads / files/guideline/01_04_shinkin.pdf (accessed on 11 April 2021).

15. Bellmann, R.; Smuszkiewicz, P. Pharmacokinetics of antifungal drugs: Practical implications for optimized treatment of patients. Infection 2017, 45, 737-779. [CrossRef]

16. Kawabe, A.; Muraki, Y.; Inose, R.; Kusama, Y.; Goto, R.; Ebisui, A.; Ishii, S.; Ishikane, M.; Ohge, H.; Ohmagari, N. Trends of Antifungal Use Based on Sales Data in Japan from 2006 to 2015. Biol. Pharm. Bull. 2020, 43, 1248-1252. [CrossRef]

17. Takakura, S.; Fujihara, N.; Saito, T.; Kudo, T.; Iinuma, Y.; Ichiyama, S. National surveillance of species distribution in blood isolates of Candida species in Japan and their susceptibility to six antifungal agents including voriconazole and micafungin. $J$. Antimicrob. Chemother. 2004, 53, 283-289. [CrossRef]

18. Kakeya, H.; Yamada, K.; Kaneko, Y.; Yanagihara, K.; Tateda, K.; Maesaki, S.; Takesue, Y.; Tomono, K.; Kadota, J.-I.; Kaku, M.; et al. National Trends in the Distribution of Candida Species Causing Candidemia in Japan from 2003 to 2014. Med. Mycol. J. 2018, 59, E19-E22. [CrossRef] [PubMed]

19. Sakagami, T.; Kawano, T.; Yamashita, K.; Yamada, E.; Fujino, N.; Kaeriyama, M.; Fukuda, Y.; Nomura, N.; Mitsuyama, J.; Suematsu, H.; et al. Antifungal susceptibility trend and analysis of resistance mechanism for Candida species isolated from bloodstream at a Japanese university hospital. J. Infect. Chemother. 2019, 25, 34-40. [CrossRef] [PubMed]

20. Pfaller, M.A.; Diekema, D.; Turnidge, J.D.; Castanheira, M.; Jones, R.N. Twenty Years of the SENTRY Antifungal Surveillance Program: Results for Candida Species From 1997-2016. Open Forum Infect. Dis. 2019, 6, S79-S94. [CrossRef] [PubMed]

21. Forrest, G.N.; Weekes, E.; Johnson, J.K. Increasing incidence of Candida parapsilosis candidemia with caspofungin usage. J. Infect. 2008, 56, 126-129. [CrossRef]

22. Perlin, D.S. Echinocandin Resistance in Candida. Clin. Infect. Dis. 2015, 61, S612-S617. [CrossRef] 
23. CLSI. Performance Standards for Antifungal Susceptibility Testing of Yeasts, 1st ed.; CLSI Supplement, M60; Wayne, P.A., Ed.; Clinical and Laboratory Standards Institute: Wayne, SA, USA, 2017; pp. 1-3. Available online: http:/ / em100.edaptivedocs.net/GetDoc. aspx?doc=CLSI\%20M60\%20ED1:2017\&xormat=SPDF\&src=BB (accessed on 11 April 2021).

24. Samura, M.; Hirose, N.; Kurata, T.; Ishii, J.; Nagumo, F.; Takada, K.; Koshioka, S.; Uchida, M.; Yamamoto, S.; Inoue, J.; et al. Support for fungal infection treatment mediated by pharmacist-led antifungal stewardship activities. J. Infect. Chemother. 2020, 26, 272-279. [CrossRef]

25. Vallabhaneni, S.; Baggs, J.; Tsay, S.; Srinivasan, A.R.; A Jernigan, J.; Jackson, B.R. Trends in antifungal use in US hospitals, $2006-12$. J. Antimicrob. Chemother. 2018, 73, 2867-2875. [CrossRef]

26. Garnacho-Montero, J.; Díaz-Martín, A.; García-Cabrera, E.; de Pipaón, M.R.P.; Hernández-Caballero, C.; Martín, J.A.; Cisneros, J.M.; Ortiz-Leyba, C. Risk Factors for Fluconazole-Resistant Candidemia. Antimicrob. Agents Chemother. 2010, 54, 3149-3154. [CrossRef] [PubMed]

27. Taplitz, R.A.; Kennedy, E.B.; Flowers, C.R. Antimicrobial Prophylaxis for Adult Patients With Cancer-Related Immunosuppression: ASCO and IDSA Clinical Practice Guideline Update Summary. J. Oncol. Pract. 2018, 14, 692-695. [CrossRef]

28. Maertens, J.A.; Girmenia, C.; Brüggemann, R.J.; Duarte, R.F.; Kibbler, C.C.; Ljungman, P.; Racil, Z.; Ribaud, P.; Slavin, M.; A Cornely, O.; et al. European guidelines for primary antifungal prophylaxis in adult haematology patients: Summary of the updated recommendations from the European Conference on Infections in Leukaemia. J. Antimicrob. Chemother. 2018, 73, 3221-3230. [CrossRef]

29. Fleming, S.; Yannakou, C.K.; Haeusler, G.; Clark, J.; Grigg, A.; Heath, C.H.; Bajel, A.; Van Hal, S.J.; Chen, S.C.; Milliken, S.T.; et al. Consensus guidelines for antifungal prophylaxis in haematological malignancy and haemopoietic stem cell transplantation, Intern. Med. J. 2014, 44, 1283-1297. [CrossRef]

30. Zonios, D.I.; Bennett, J.E. Update on Azole Antifungals. Semin. Respir. Crit. Care Med. 2008, 29, 198-210. [CrossRef]

31. Matsumoto, K.; Ikawa, K.; Abematsu, K.; Fukunaga, N.; Nishida, K.; Fukamizu, T.; Shimodozono, Y.; Morikawa, N.; Takeda, Y.; Yamada, K. Correlation between voriconazole trough plasma concentration and hepatotoxicity in patients with different CYP2C19 genotypes. Int. J. Antimicrob. Agents 2009, 34, 91-94. [CrossRef]

32. Arendrup, M.C.; Sulim, S.; Holm, A.; Nielsen, L.; Nielsen, S.D.; Knudsen, J.D.; Drenck, N.E.; Christensen, J.J.; Johansen, H.K. Diagnostic Issues, Clinical Characteristics, and Outcomes for Patients with Fungemia. J. Clin. Microbiol. 2011, 49, 3300-3308. [CrossRef] [PubMed]

33. Bizerra, F.C.; Jimenez-Ortigosa, C.; Souza, A.C.R.; Breda, G.L.; Queiroz-Telles, F.; Perlin, D.; Colombo, A.L. Breakthrough Candidemia Due to Multidrug-Resistant Candida glabrata during Prophylaxis with a Low Dose of Micafungin. Antimicrob. Agents Chemother. 2014, 58, 2438-2440. [CrossRef]

34. Andes, D.R.; Diekema, D.; Pfaller, M.; Marchillo, K.; Bohrmueller, J. In Vivo Pharmacodynamic Target Investigation for Micafungin against Candida albicans and C. glabrata in a Neutropenic Murine Candidiasis Model. Antimicrob. Agents Chemother. 2008, 52, 3497-3503. [CrossRef] [PubMed]

35. Almirante, B.; Rodríguez, D.; Cuenca-Estrella, M.; Almela, M.; Sanchez-Reus, F.; Ayats, J.; Alonso-Tarres, C.; Rodriguez-Tudela, J.L.; Pahissa, A.; The Barcelona Candidemia Project Study Group. Epidemiology, Risk Factors, and Prognosis of Candida parapsilosis Bloodstream Infections: Case-Control Population-Based Surveillance Study of Patients in Barcelona, Spain, from 2002 to 2003. Clin. Microbiol. 2006, 44, 1681-1685. [CrossRef] [PubMed]

36. Kato, H.; Yoshimura, Y.; Suido, Y.; Shimizu, H.; Ide, K.; Sugiyama, Y.; Matsuno, K.; Nakajima, H. Mortality and risk factor analysis for Candida blood stream infection: A multicenter study. J. Infect. Chemother. 2019, 25, 341-345. [CrossRef] [PubMed]

37. Andes, D.; Ambrose, P.G.; Hammel, J.P.; Van Wart, S.A.; Iyer, V.; Reynolds, D.K.; Buell, D.N.; Kovanda, L.L.; Bhavnani, S.M. Use of Pharmacokinetic-Pharmacodynamic Analyses To Optimize Therapy with the Systemic Antifungal Micafungin for Invasive Candidiasis or Candidemia. Antimicrob. Agents Chemother. 2011, 55, 2113-2121. [CrossRef]

38. Kapralos, I.; Mainas, E.; Neroutsos, E.; Apostolidi, S.; Siopi, M.; Apostolopoulou, O.; Dimopoulos, G.; Sambatakou, H.; Valsami, G.; Meletiadis, J.; et al. Population pharmacokinetics of micafungin over repeated doses in critically ill patients: A need for a loading dose? J. Pharm. Pharmacol. 2020, 72, 1750-1760. [CrossRef]

39. Burton, M.J.; Swiatlo, E.; Shah, P. Misidentification of Candida parapsilosis as C. famata in a Clinical Case of Vertebral Osteomyelitis. Am. J. Med. Sci. 2011, 341, 71-73. [CrossRef]

40. Castanheira, M.; Woosley, L.; Diekema, D.; Jones, R.N.; Pfaller, M. Candida guilliermondii and Other Species of Candida Misidentified as Candida famata: Assessment by Vitek 2, DNA Sequencing Analysis, and Matrix-Assisted Laser Desorption Ionization-Time of Flight Mass Spectrometry in Two Global Antifungal Surveillance Programs. J. Clin. Microbiol. 2012, 51, 117-124. [CrossRef]

41. Kim, S.H.; Shin, J.H.; Mok, J.H.; Kim, S.Y.; Song, S.A.; Kim, H.R.; Kook, J.-K.; Chang, Y.-H.; Bae, I.K.; Lee, K. Misidentification of Candida guilliermondii as C. famata among Strains Isolated from Blood Cultures by the VITEK 2 System. Biomed. Res. Int. 2014, 2014, 250408. [CrossRef]

42. Huang, Y.-S.; Wang, F.-D.; Chen, Y.-C.; Hsieh, M.-H.; Hii, I.-M.; Lee, Y.-L.; Ho, M.-W.; Liu, C.-E.; Chen, Y.-H.; Liu, W.-L. High rates of misidentification of uncommon Candida species causing bloodstream infections using conventional phenotypic methods. $J$. Formos. Med. Assoc. 2021, 120, 1179-1187. [CrossRef] 